\title{
Convolutional Neural Networks for Leaf Disease Classification
}

\author{
Raí G. Carvalho, Leticia T. M. Zoby \\ Ciência da Computação - Instituto de Educação Superior de Brasília (IESB) \\ Brasília, DF - Brazil \\ raigc20@gmail.com, letmaia@gmail.com
}

\begin{abstract}
This paper aims to improve the classification process of leaf diseases in plantations, reducing the need to have a specialist or prior knowledge of the diseases that can affect a plantation, since some diseases can spread and end with entire plantations. The proposal is the use of Convolutional Neural Networks (CNN) to classify leaf diseases in plants using images, creating a model that can be implemented in a smartphone application. The model selected for the application, using a dataset with 4485 images separated in 5 classes, had an accuracy of $97 \%$ in the test base.
\end{abstract}

Resumo. Este artigo busca aprimorar o processo de classificação de doenças foliares em plantações, reduzindo a necessidade de ter um especialista ou um conhecimento prévio das doenças que podem afetar uma plantação, visto que algumas doenças podem se espalhar e acabar com plantações inteiras. A proposta é a utilização de Redes Neurais Convolucionais (RNC) para a classificação de doenças foliares em plantas por meio de imagens, gerando um modelo que possa ser implementado em uma aplicação para smartphones. $O$ modelo selecionado para a aplicação, usando uma base de dados de 4485 imagens separadas em 5 classes, teve uma acurácia de 97\% na base de testes.

\section{Introdução}

A agricultura é parte essencial da economia brasileira, sendo um dos maiores produtores agrícolas do mundo segundo relatório disponibilizado pelo United States Department of Agriculture - Departamento de Agricultura dos Estados Unidos [USDA 2017]. Segundo último censo agropecuário realizado pelo IBGE, $84 \%$ dos estabelecimentos agropecuários sendo de agricultura familiar [IBGE 2006]. Tendo em vista que perdas na agricultura devido a doenças e pragas são um dos maiores perigos enfrentados pelos agricultores, são necessários métodos mais acessíveis para a identificação dessas doenças [Cerda 2017].

Um dos grandes desafios na agricultura está em diagnosticar corretamente doenças causadas por fitopatógenos, já que diferentes doenças podem ter sintomas semelhantes e o tratamento errado pode agravar a situação da planta. Outro fator crucial é o tempo de identificação da doença, visto que a demora do tratamento pode levar a contaminação de outras plantas e gerar perdas muito maiores [Kreyci and Menten 2013].

É importante também considerar o impacto que perdas na agricultura podem causar em outros setores da economia. Em um estudo de caso, realizado em uma empresa de bioenergia, concluiu que o as perdas em uma plantação de cana-de-açúcar resultaram em um prejuízo de 2,8 milhões para a empresa [Zuza and Borsato 2013]. 
Outro estudo apontou que a redução de perdas na agricultura no Brasil para níveis similares ao de países desenvolvidos resultaria em um aumento do PIB em 9,7 bilhões e a criação de mais de 300 mil empregos [Costa et al. 2015].

Embora existam manuais que possam auxiliar agricultores na classificação de doenças em plantas [Garrido and Gava 2014], isso ainda requer um trabalho repetitivo e demorado em que um erro pode resultar na perda da planta, e a utilização de especialistas gera mais gastos e em casos de pequenos agricultores pode não ser uma opção viável.

A utilização de técnicas de aprendizado de máquinas para a classificação de plantas a partir de descritores morfológicos como folhas, por exemplo, possuem considerável abundância na literatura em consequência da contribuição importante que o reconhecimento de espécimes vegetais, por sistemas computacionais, oferece ao setor agronômico e computacional [Sousa et al. 2017].

De acordo com Sousa [Sousa et al. 2017 apud Gu et al. 2015, Jassmann et al. 2015] Redes Neurais Convolucionais - RNC (ou, em inglês, Convolutional Neural Networks - CNNs) são consideradas um modelo matemático de classificação inspirado no sistema biológico de processamento de dados visuais. Na literatura, esses algoritmos dispõem de resultados satisfatórios para uma diversidade de problemas, incluindo classificação vegetal.

O trabalho tem como objetivo apresentar uma aplicação móvel integrada a uma Rede Neural Convolucionais (RNC) para realizar a classificação das doenças foliares e assim auxiliar agricultores. Para esse trabalho houve um treinamento de uma RNA para a classificação de doenças foliares, especificamente folhas de videiras.

Este trabalho está dividido da seguinte forma: a seção 2 aborda os trabalhos correlatos; na seção 3 é apresentada a metodologia; a seção 4 apresenta os resultados; e por fim, na seção 5 são apresentadas as conclusões e os trabalhos futuros.

\section{Trabalhos Correlatos}

Considerando que nos últimos anos os avanços na área de visão computacional vêm sendo significativos, especialmente desde a performance obtida por uma Rede Neural Convolucional (RNC) para a competição realizada pelo Image-Net LSVRC-2010 [Krizhevsky et al. 2012], neste trabalho é proposto o treinamento de uma RNC para a classificação de doenças em plantas com base nos sintomas apresentados nas folhas e a integração do modelo a uma aplicação Android para classificação em tempo real.

Em [Sladojevic et al. 2016], é proposto um método de detecção de doenças foliares utilizando deep learning em conjunto com RNC, capaz de detectar 13 diferentes doenças em plantas e com uma precisão de 96,3\% utilizando-se de uma base de dados com mais de 30000 imagens. O trabalho sugere o uso de técnicas de transformações de imagens para obter uma quantidade de dados ideal para treino além do uso de uma classe de fundo ou background para ajudar o modelo a distinguir as folhas do ambiente ao fundo da imagem.

Um modelo de solução por meio do processamento de imagens que seja apropriado para a implementação em smartphones, proposto por [Petrellis 2015], conta com a comunicação com um banco de dados na nuvem e o uso de sensores para testes mais precisos e, utilizando o Spot Recognition System (SRS) desenvolvido na pesquisa, obteve uma precisão acima de $90 \%$. 
Para a classificação de cultivares de guaranazeiros, [Sousa et al. 2017] desenvolveu um sistema chamado de WAVA onde a imagem retirada por um smartphone é enviada para um servidor onde é classificada por uma RNC e o resultado é então entregue ao usuário. Foram usadas 2800 imagens, obtidas por meio de data augmentation, e o resultado foi uma precisão de $97 \%$.

Para detecção de ervas daninhas em lavouras de soja por meio de imagens tiradas por drones, [Ferreira 2017] propõe a utilização de RNC. Utilizando o software Pynovisão para a segmentação, extração de atributos e classificação da imagem, foi comparado o desempenho utilizando Redes Neurais Convolucionais, Florestas Aleatórias, C4.5, AdaBoost e Máquinas de Vetores de Suporte. Os resultados apontam uma performance melhor para as Redes Neurais Convolucionais com precisão superior a 98\%, sem a necessidade de utilizar todas as 15 mil imagens para treinar a rede.

\section{Metodologia}

\subsection{Base de dados}

Foi utilizada a base de dados do site [PlantVillage 2017], uma comunidade aberta de perguntas e respostas para auxiliar pessoas a cultivar plantas. A base possui 38 classes diferentes distribuídas entre diversas plantas, e dessas, foram utilizadas aproximadamente 4000 imagens de folhas de videiras distribuídas nas seguintes classes de doenças:

- Saudável (423 imagens) - folhas de videiras sem qualquer tipo de doença.

- Black rot (1180 imagens) - Causada pelo fungo ascomycetous fungus, comum em tempos quentes e úmidos, causa lesões escuras na planta que podem ser abertas pelo vento e em casos mais graves pode matar a planta. Embora seja originaria da América do Norte, hoje ela já afeta a América do Sul, Ásia e Europa.

- Esca (1383 imagens) - Causada pelo fungo phaeomoniella chlamydospora, mais comum na Europa, causa pequenas manchas escuras na folha da planta que podem levar a rachaduras.

- Leaf Blight ou Isariopsis (1076 imagens) - Causada pelo fungo Isariopsis clavispora, provoca a queda das folhas e reduz a produção no ano seguinte.

- Outros (423 imagens) - Imagens aleatórias retiradas da internet. doenças.

A figura 1 mostra exemplos de imagens utilizadas em cada uma das classes de 


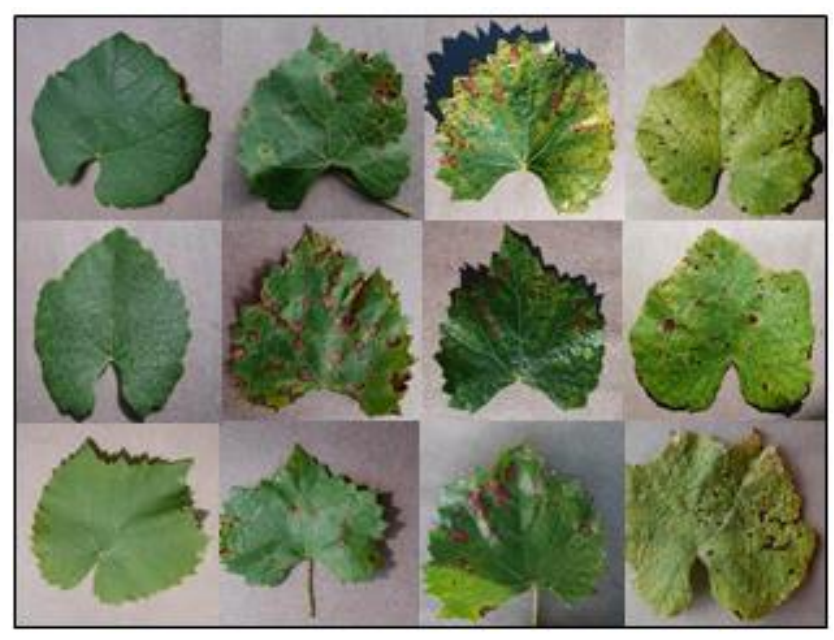

Figura 1. Exemplos de imagens da base de dados para cada classe da folha de videira, separadas por coluna da direita para esquerda: Saudável, Black rot, Esca e Isariopsis.

Foram também criadas duas outras bases de dados para a comparação de resultados. Uma delas na qual o fundo é completamente preto, chamada de bg_black, e outra onde foi utilizado tons de verde no fundo, de forma a simular um ambiente real, chamada de $b g \_$green. A base de dados original foi chamada de $b g$ _original. Na figura 2 é apresentado um exemplo de cada uma das bases.

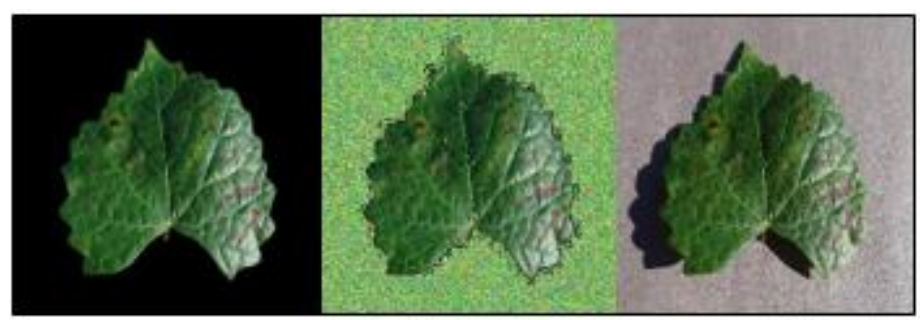

Figura 2. Exemplo de imagens para cada base de dados. Da esquerda para direita: bg_black, bg_green e bg_original.

Todas as imagens são redimensionadas para o tamanho $128 \times 128$ utilizando a biblioteca OpenCV2 em Python.

\subsection{Treinamento}

Para o treinamento foi utilizado a biblioteca do [Tensorflow 2017] para a criação da RNC composta de cinco camadas intermediarias.

As primeiras duas camadas, fazem a operação de convolução utilizando respectivamente, 32 filtros de $32 \times 32$ e 64 filtros de 64x64, e a função de ativação ReLU (Rectified Linear Unit). É também feito a operação de pooling máximo (maximum pooling) em ambas as camadas.

Na próxima camada, chamada de flatten layer é feito a redução de dimensão da saída que é então passada para duas camadas completamente conectada (Fully Conected Layer) na qual a primeira utiliza a função de ativação ReLU. Ao fim, é utilizado a função Softmax para gerar a saída como uma probabilidade entre 0 e 1 . Na figura 3 é apresentada a arquitetura da RNC. 


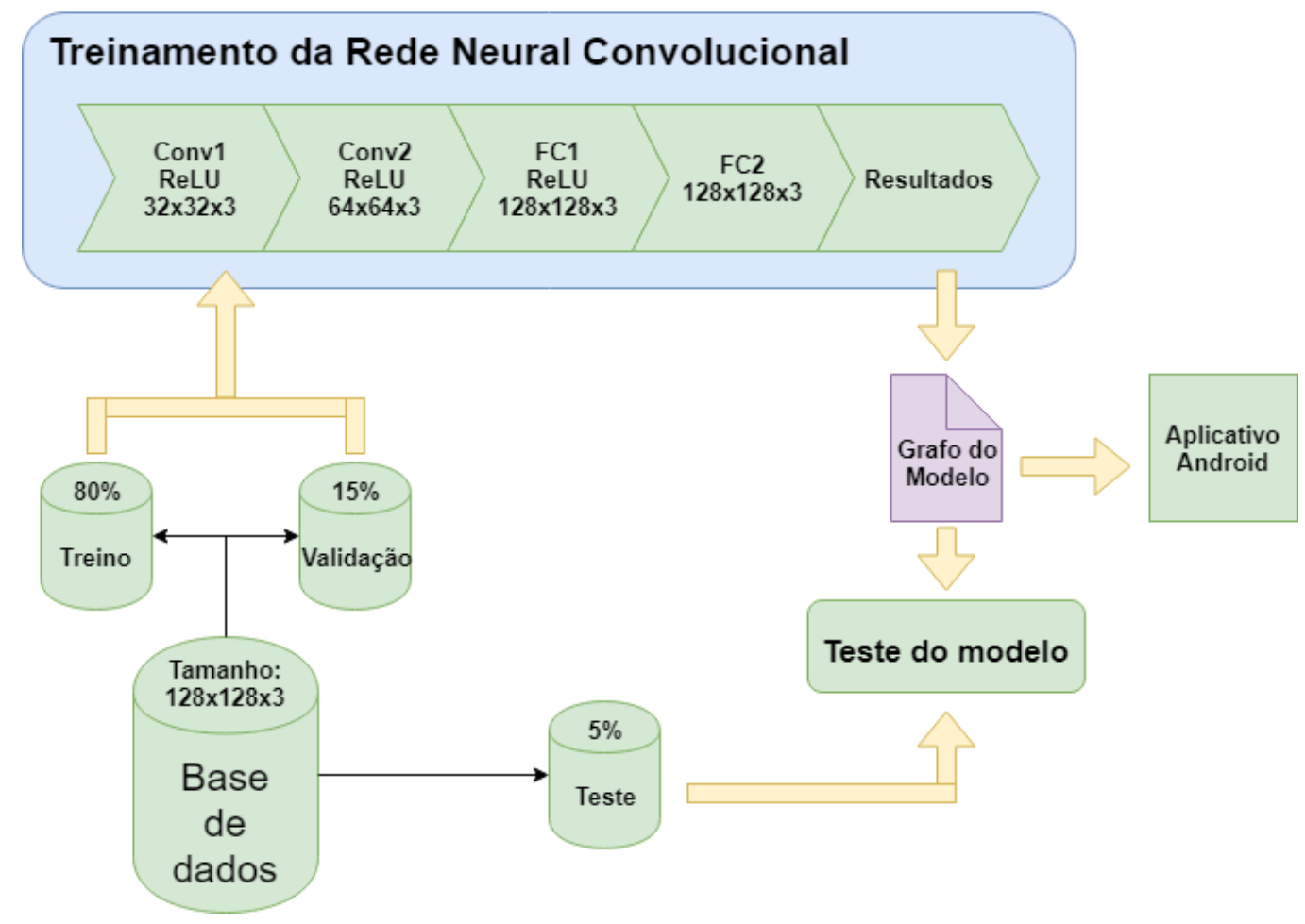

Figura 3. Arquitetura da Rede Neural Convolucional (RNC) proposta.

No total foram realizadas 150 epochs, e foi utilizado para a otimização o algoritmo Adam [Kingma and Ba 2015] devido a sua eficiência e simples implementação, com taxa de aprendizado (learning rate) de 0,0001. O treinamento foi realizado utilizando uma placa de vídeo da Nvidia GTX 980.

\subsection{Aplicação móvel}

A aplicação para dispositivos Android foi feita utilizando o exemplo disponibilizado pelo Tensorflow. $\mathrm{O}$ aplicativo funciona carregando um arquivo com o grafo do modelo treinado previamente juntamente com um arquivo de texto com o nome de cada classe, utilizando a biblioteca Android do Tensorflow para realizar a classificação em tempo real da imagem da câmera do aparelho. Caso seja necessário testar outros modelos, basta alterar o arquivo a ser carregado. A figura 4 mostra um diagrama de como funciona a aplicação. 


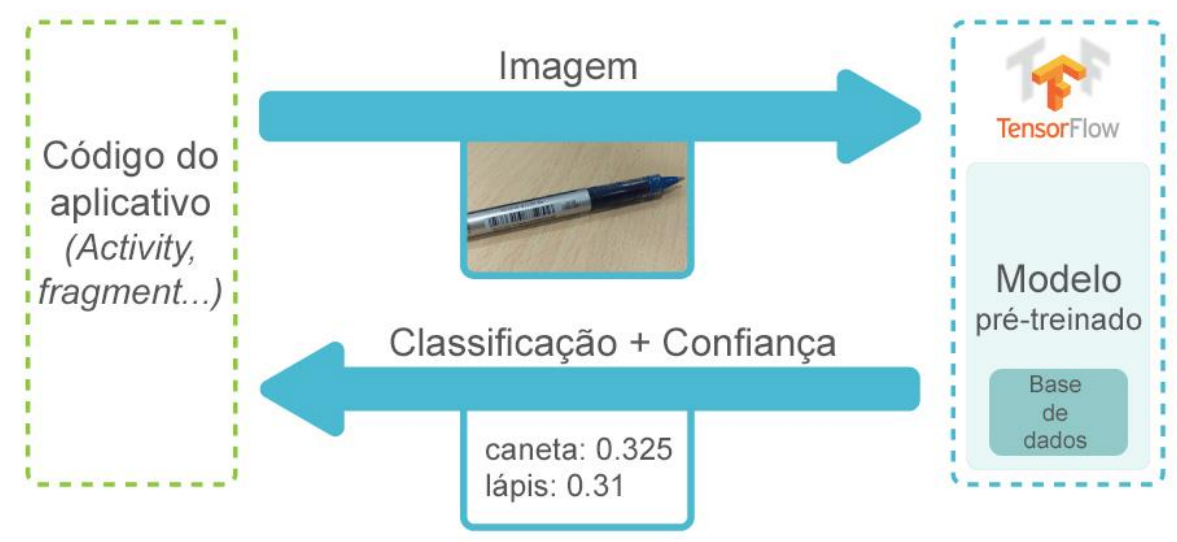

Figura 4. Estrutura geral da comunicação de aplicações Android com o Tensorflow. Adaptado de [Alammar 2016].

Para gerar o arquivo apropriado para o uso do Tensorflow no Android são utilizados os scripts em Python optmize_for_inference.py e quantize_graph.py disponibilizados pelo Tensorflow, sendo o primeiro para gerar uma versão simplificada do modelo sem o uso de operações indisponíveis na biblioteca do Android, e o segundo utilizado para reduzir o tamanho do modelo e consequentemente da aplicação.

\section{Resultados}

Nesta seção são apresentados os resultados obtidos na metodologia proposta em classificar as imagens das folhas de videiras em Black rot; Esca; Leaf Blight ou Isariopsis; ou Saudáveis, com base nas imagens de teste.

Dentre as 4485 imagens utilizadas, $15 \%$ delas foram utilizadas para constituir a base de validação e 5\% para a base de testes. Os $80 \%$ restantes foram utilizados para o treinamento da rede.

Foram realizados testes alterando o tamanho dos batches e dos filtros utilizados na RNC, e os resultados apresentados na tabela 1 e no gráfico da figura 5. 
Tabela 1. Resultados dos modelos treinados.

\begin{tabular}{|c|c|c|c|c|c|c|}
\hline Modelo & $\begin{array}{c}\text { Base de } \\
\text { dados }\end{array}$ & $\begin{array}{c}\text { Tamanho } \\
\text { dos batchs }\end{array}$ & $\begin{array}{c}\text { Tamanho } \\
\text { dos filtros }\end{array}$ & $\begin{array}{c}\text { Acurácia } \\
\text { na base } \\
\text { de } \\
\text { validação } \\
\text { (Val_acc } \\
\text { (Val_loss })\end{array}$ & $\begin{array}{c}\text { Perda na } \\
\text { base de } \\
\text { validação } \\
(\text { Valest_acc })\end{array}$ & $\begin{array}{c}\text { Acurácia } \\
\text { na base de } \\
\text { testes } \\
\text { (Tes_an }\end{array}$ \\
\hline $\begin{array}{c}\text { cnn-128- } \\
\text { model-0 }\end{array}$ & bg_green & 32 & $32-64-128$ & 0,9062 & 0,5050 & 0,97 \\
\hline $\begin{array}{c}\text { cnn-128- } \\
\text { model-1 }\end{array}$ & bg_black & 32 & $32-64-128$ & 0,9375 & 0,4926 & 0,94 \\
\hline $\begin{array}{c}\text { cnn-128- } \\
\text { model-2 }\end{array}$ & bg_original & 32 & $32-64-128$ & 0,9687 & 0,4499 & 0,97 \\
\hline $\begin{array}{c}\text { cnn-128- } \\
\text { model-3 }\end{array}$ & bg_green & 20 & $32-64-128$ & 1,0 & 0,0009 & 0,94 \\
\hline $\begin{array}{c}\text { cnn-128- } \\
\text { model-4 }\end{array}$ & bg_black & 20 & $32-64-128$ & 0,8999 & 0,7450 & 0,95 \\
\hline $\begin{array}{c}\text { cnn-128- } \\
\text { model-5 }\end{array}$ & bg_original & 20 & $32-64-128$ & 1,0 & 0,0009 & 0,96 \\
\hline $\begin{array}{c}\text { cnn-128- } \\
\text { model-6 }\end{array}$ & bg_green & 32 & $64-64-128$ & 0,8750 & 0,4345 & 0,95 \\
\hline $\begin{array}{c}\text { cnn-128- } \\
\text { model-7 }\end{array}$ & bg_black & 32 & $64-64-128$ & 0,9062 & 0,1798 & 0,94 \\
\hline $\begin{array}{c}\text { cnn-128- } \\
\text { model-8 }\end{array}$ & bg_original & 32 & $64-64-128$ & 0,9687 & 0,6208 & 0,97 \\
\hline
\end{tabular}

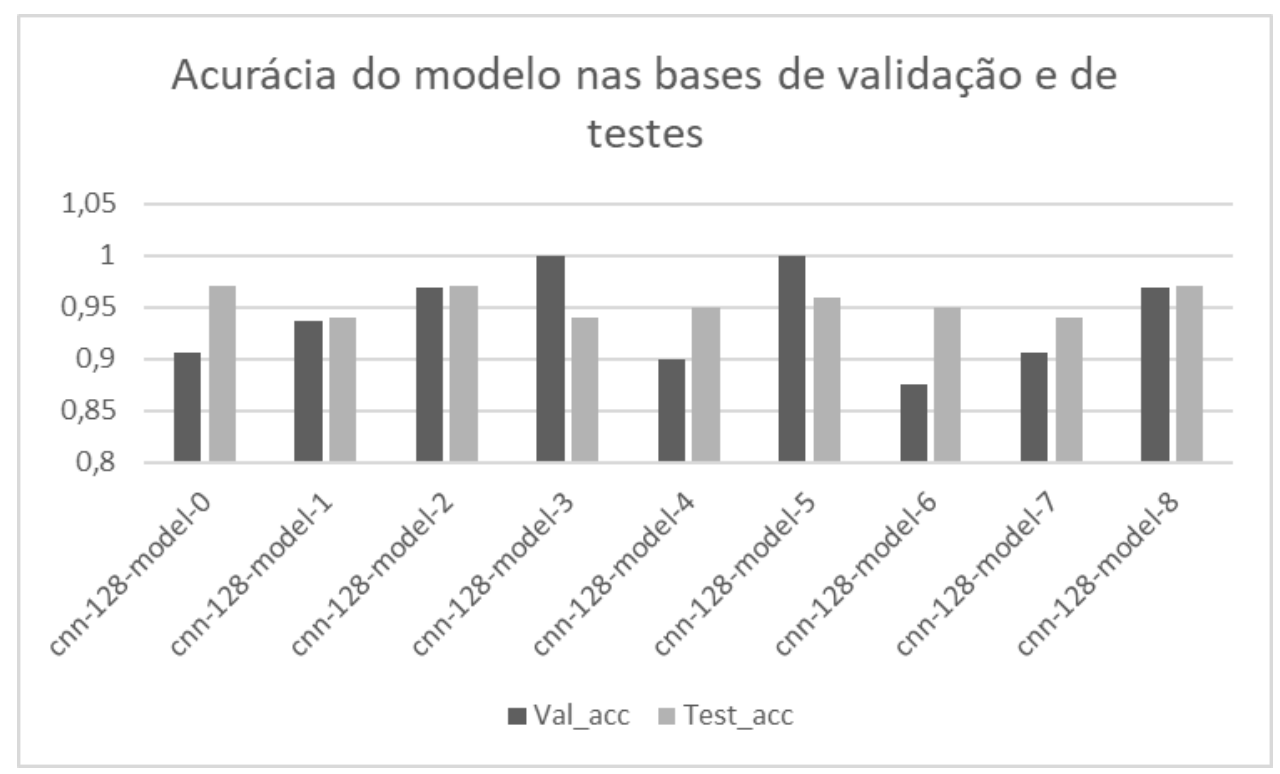

Figura 5. Gráfico da acurácia dos modelos para bases de validação e teste. 
Foi observado que os modelos com melhores resultados foram aqueles utilizando a base de dados original (bg_original), obtendo uma acurácia máxima de 97\%. Durante os testes foi também constatado um pior desempenho na classificação da doença Black rot, chegando a ser responsável a aproximadamente $40 \%$ das classificações incorretas. Por fim, foi selecionado o modelo cnn-128-model-2 para ser implementado na aplicação.

Na tabela 2 é apresentada a comparação do modelo selecionado, cnn-128-model2, com os modelos do MobileNet-V2, Inception-V3, ResNet-V2 e NASNet-A na tarefa de classificação das imagens. Tais modelos foram treinados na base de dados da competição realizada pelo ImageNet [ILSVRC 2012] e utilizando a técnica de transfer learning foi feito o treinamento para a classificação das doenças foliares.

Tabela 2. Comparação do modelo selecionado e modelos populares.

\begin{tabular}{|c|c|c|c|}
\hline Modelos & $\begin{array}{c}\text { Acurácia na base } \\
\text { de validação } \\
(\text { Val_acc })\end{array}$ & $\begin{array}{c}\text { Perda na base de } \\
\text { validação } \\
(\text { Val_loss })\end{array}$ & $\begin{array}{c}\text { Acurácia na base } \\
\text { de testes (Test_acc) }\end{array}$ \\
\hline Cnn-128-model-2 & 0,9687 & 0,4499 & 0,97 \\
\hline MobileNet-V2 & 0,9380 & 0,2257 & 0,95 \\
\hline Inception-V3 & 0,9690 & 0,5985 & 0,91 \\
\hline ResNet-V2 & 0,9690 & 0,2676 & 0,91 \\
\hline NASNet-A & 0,9380 & 0,4399 & 0,91 \\
\hline
\end{tabular}

Dentre os modelos usados para comparação, o melhor resultado na base de testes foi obtido pelo MobileNet-V2 com uma acurácia de 95\%, enquanto o Inception-V3, ResNet-V2 e o NASNet-A tiveram uma acurácia de 91\%. Ainda assim, todos os modelos comparados tiveram um resultado inferior ao modelo treinado com uma acurácia de $97 \%$. Em todos os modelos usados para comparação foram utilizados os mesmos parâmetros do modelo cnn-128-model-2.

Uma possível causa para os resultados inferiores nos modelos comparados é a alta complexidade de suas arquiteturas, que em um problema com poucas classes e imagens para treinamento e uma complexidade baixa, pode acabar sendo prejudicial para o aprendizado da rede. Com um maior número de plantas e doenças para serem classificadas, modelos mais complexos podem ser necessários e os modelos utilizados para comparação podem obter um melhor desempenho.

A aplicação desenvolvida utilizando o modelo treinado consiste de uma única tela onde é apresentado a classificação e a probabilidade de pertencer a ela na parte superior da tela, enquanto o espaço restante é utilizado pela câmera do aparelho. As três classes com maior probabilidade são apresentadas, sendo necessário uma probabilidade mínima de 0,1 para que seja apresentada. Na figura 6 é mostrada a interface da aplicação. 


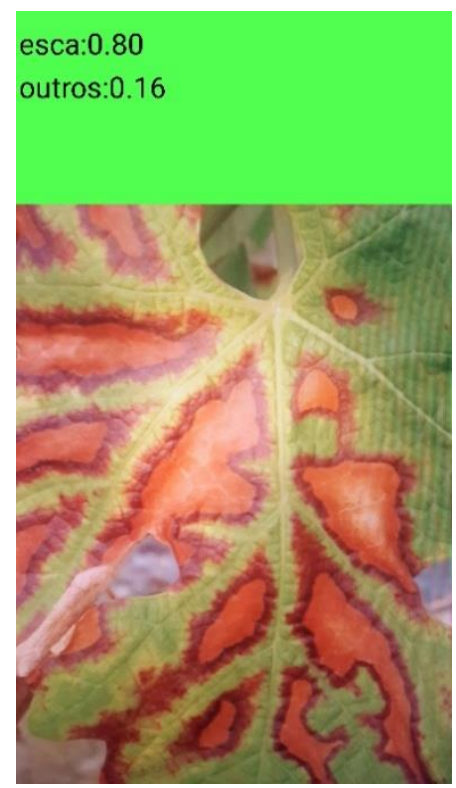

Figura 6. Tela da aplicação para classificar doenças foliares em plantas.

\section{Conclusão e Trabalhos Futuros}

O modelo selecionado, cnn-128-model-2, obteve uma acurácia de 96,87\% na base de validação e $97 \%$ para a base de testes. A base de dados com melhores resultados foi a bg_original, contra a intuição de que o fundo preto dando destaque a folha pudesse ajudar a classificação durante o treinamento para melhores resultados. Foi observado também que a classe de doença Black rot apresentou resultados inferiores na classificação das imagens de teste, chegando a ser responsável por aproximadamente $40 \%$ das classificações erradas durante os testes.

$\mathrm{Na}$ comparação com modelos do MobileNet-V2, Inception-V3, ResNet-V2 e NASNet-A, treinados na base de dados do ImageNet e utilizando a técnica de transfer learning para treinar a rede para a classificação das doenças foliares, o modelo treinado mostrou um resultado superior na base de testes. Com uma base de dados maior e a adição de novas plantas, os modelos mais complexos serão necessários para um melhor desempenho.

A aplicação Android não pôde ser testada em ambiente real devido à dificuldade do acesso a plantações de videiras que possuem as doenças específicas disponíveis na base de dados. A expectativa é de que o modelo utilizando o fundo verde possa se sair melhor em situações de classificação em ambiente real, porém é também possível indicar que o usuário coloque uma folha branca ou preta ao fundo da folha da planta no momento da classificação, podendo gerar resultados melhores nos modelos da base de dados original e das imagens com fundo preto.

Para trabalhos futuros, o uso ou criação de uma base de dados maior e mais adequada, isto é, com imagens da folha ainda na planta para que facilite a detecção em uma situação real, tornar mais prático a alteração do modelo utilizado, podendo ser feito pelo próprio usuário da aplicação, e adicionar novas classes de doenças e plantas, bem como informações sobre tratamento, para que possa ajudar agricultores de diversos tipos de plantas. 


\section{Referências}

Alammar, J. (2016), https://jalammar.github.io/visual-interactive-guide-basics-neuralnetworks/

Cerda, R., Avelino, J., Gary, C., et al. (2017) "Primary and Secondary Yield Losses Caused by Pests and Diseases: Assessment and Modeling in Coffee", In: GAIA.

Costa, C. C., Guilhoto, J. J. M. and Burnquist, H. L. (2015) "Impactos Socioeconômicos de Reduções nas Perdas Pós-colheita de Produtos Agrícolas no Brasil”, In: RESR, v. 53, p. 395-408.

Ferreira, S. (2017) "Redes Neurais Convolucionais Profundas na Detecção de Plantas Daninhas em Lavoura de Soja", In: UFMG.

Garrido, L. R. and Gava, R. (2014) "Manual de doenças fúngicas da videira”, In: Embrapa.

Gu, J., Wang, Z., Kuen, J., Ma, L., Shahroudy, A., Shuai, B., Liu, T., Wang, X., and Wang, G. (2015). "Recent advances in convolutional neural networks", In: arXiv preprint arXiv:1512.07108.

IBGE (2006) “Censo Agropecuário", In: Instituto Brasileiro de Geografia e Estatística, https://biblioteca.ibge.gov.br/visualizacao/periodicos/50/agro_2006_agricultura_fami liar.pdf

ILSVRC (2012) “ImageNet Large Scale Visual Recognition Challenge”, In: ImageNet, http://image-net.org/challenges/LSVRC/2012/index

Jassmann, T. J., Tashakkori, R., and Parry, R. M. (2015) "Leaf classification utilizing a convolutional neural network", In SoutheastCon 2015, pages 1-3. IEEE.

Kingma, D. P. and Ba, J. L. (2015) “Adam: A Method for Stochastic Optimization”, In: ICLR.

Kreyci, P. F. and Menten, J. O. (2013) "Limitadoras de produtividade", In: Cultivar Caderno Técnico, v. 167, p. 1-12.

Krizhevsky, A., Sutskever, I. and Geoffrey, E. H. (2012) "ImageNet Classification with Deep Convolutional Neural Networks", In: NIPS, p. 1-9.

Petrellis, N. (2015) "Plant Disease Diagnosis Based on Image Processing, Appropriate for Mobile Phone Implementation", In: HAICTA, p. 238-246.

PlantVillage (2017), https://plantvillage.psu.edu/

Sladojevic, S., Arsenovic, M., Anderla, A., Culibrk, D. and Stefanovic, D. (2016) "Deep Neural Networks Based Recognition of Plant Diseases by Leaf Image Classification", In: Computational Intelligence and Neuroscience, v. 2016, p. 11.

Sousa, A. L., Salame, M. F. A., Nascimento Filho, F. J. and Atroch, A. L. (2017) "Redes Neurais Convolucionais Aplicadas ao Processo de Classificação de Cultivares de Guaranazeiros", In: XIV Encontro Nacional de Inteligência Artificial e Computacional

Tensorflow (2017), https://www.tensorflow.org/

USDA (2017) "World Agricultural Production", In: Circular Series, http://apps.fas.usda.gov/psdonline/circulars/production.pdf 
Zuza, J. L. M. F. and Borsato, J. M. S. L. (2013) "Análise de oerdas agrícolas e econômicas ocasionada pela doença ferrugem alaranjada em canaviais comerciais de uma empresa de bioenergia no interior de São Paulo", In: XX Congresso Brasileiro de Custos, p. 13. 